\title{
【研究简报】
}

\section{Maslov 型迭代指标的一个最佳增长估计}

\author{
刘春根 龙以明 \\ (南开数学研究所, 天津 300071)
}

\section{关键词 Maslov型指标 Maslov 型平均指标 迭代不等式}

在讨论线性和非线性 Hamilton 系统的解的存在性、多重性和稳定性问题时, Maslov 型指 标理论起到关键性的作用. 考虑一个线性 Hamilton 系统

$$
\dot{y}=J B(t) y, y \in \mathbb{R}^{2 n} .
$$

这里 $J=\left(\begin{array}{rr}0 & -I \\ I & 0\end{array}\right), I$ 是 $\mathbb{R}^{n}$ 中的恒等矩阵, $B(t)$ 是一个对称的, 以 $T>0$ 为周期的矩阵, 它关于 时间 $t$ 连续, 即 $B \in C\left(S_{T}, \mathscr{L}_{s}\left(\mathbb{R}^{2 n}\right)\right), S_{T}=\mathbb{R} /(T \mathbb{Z}), \mathscr{L}_{s}\left(\mathbb{R}^{2 n}\right)$ 是 $2 n \times 2 n$ 对称实矩阵的集合. 系统 (1) 的基本解 $\gamma(t)$ 给出辛群中由恒等矩阵出发的一条道路. Conley 和 Zehnder 在他们 1984 年的论文 ${ }^{[1]}$ 中, 建立了当 $n \geqslant 2$ 时非退化辛道路的 Maslov 型指标. 本文第二作者和 Zehnder 在 1990 年的论文 ${ }^{[2]}$ 中, 把非退化辛道路的 Maslov 型指标理论推广到 $n=1$ 的情形. 而退化辛道路的 Maslov 型指标理论则是由本文第二作者在文献 $[3,4]$ 和最近的文章 ${ }^{1)}$ 中建立 的. 对于线性 Hamilton 系统 (1) 来说, 它的 Maslov 型指标是由它的基本解 (作为一条辛道路) 所确定的一对数 $\left(i_{T}(B), \nu_{T}(B)\right) \in \mathbb{Z} \times\{0, \cdots, 2 n\}$. 这里 $i_{T}(B)$ 是其指标部分, 而 $\nu_{T}(B)$ 则 是其零维数.

如果 $(x, T)$ 是下述非线性 Hamilton 系统的 $T$ - 周期解:

$$
\dot{x}(t)=J H^{\prime}(t, x(t)), x(t) \in \mathbb{R}^{2 n} .
$$

这里 $H \in C^{1}\left(\mathbb{R} \times \mathbb{R}^{2 n}, \mathbb{R}\right)$ 并且它关于时间 $t$ 是 $T$-周期函数. 式 (2) 的线性化是下述线性 Hamilton 系统

$$
\dot{y}=J H^{\prime \prime}(t, x(t)) y, y \in \mathbb{R}^{2 n} .
$$

这时把方程 (3) 的 Maslov 型指标叫做 $x$ 的 Maslov 型指标, 记为 $\left(i_{T}(x), \nu_{T}(x)\right)$. 如果把 $B(t)$ 看作是一个 $k T$ - 周期 ( $k \in \mathbb{N}$ 即自然数集) 的矩阵函数, 就得到相应的 $\left(i_{k T}(B), \nu_{k T}(B)\right)$, 或者把 $x$ 看作是一个 $k T$ - 周期的解 $x^{k}$ (通过迭代延拓), 就得到相应的 $\left(i_{k T}\left(x^{k}\right), \nu_{k T}\left(x^{k}\right)\right.$ ). 以

\section{参考文献}

1 Knüppel F, Nielsen K. SL(V) is 4-reflectional. Geometria Dedicata, 1991, 88: 301 308

2 Hahn A. Algebraic K-theory, Morita theory and the classical groups. In: Lecture Notes in Mathematics, 1984, 1185: 88 117

3 Silvester J R. Introduction to Algebraic K-theory. London, New York: Chapman and Hall, 1981

4 Sourour A R. A factorization theorem for matrices, Lin and Multilin. Algebra, 1986, 19: 141 147

$(1997-02-19$ 收稿 $)$

1) Long Y. A Maslov-type index theory for symplectic paths. Preprint series of Nankai Inst of Math, 1997 
下省去此记号中的 $B$ 和 $x^{k}$, 序列 $\left\{\left(i_{k T}, \nu_{k T}\right)\right\}_{k \in N}$ 包含着比 $\left(i_{T}, \nu_{T}\right)$ 更丰富的信息. 文献 [5] 给出了 $i_{k T}, k \in \mathbb{N}$ 的一个估计式, 本文利用这一估计给出 $i_{k T}, \forall k \in \mathbb{N}$ 的一个最佳增长不等 式.

定义 称下述极限

$$
\hat{i}_{T}=\lim _{k \rightarrow+\infty} \frac{i_{k T}}{k}
$$

为关于周期 $T$ 的平均 Maslov 型指标.

在 Long 文 ${ }^{1)}$ 中, 证明了这一极限存在(是一个有限实数).下面是本文的主要结果.

定理 对所有的 $k \in \mathbb{N}$, 有

$$
k \hat{i}_{T}-n \leqslant i_{k T} \leqslant k \hat{i}_{T}+n-\nu_{k T} .
$$

证 由文献[5]中的定理 4.1 , 对 $k \in \mathbb{N}$, 若 $\nu_{k T}=0$, 有 $\nu_{T}=0$ 并且

$$
\begin{aligned}
i_{k T}= & k\left(i_{T}-\mu\right)+\sum_{j=1}^{n} 2 t_{j}+\mu \geqslant \\
& k\left(i_{T}-n\right)+n=k\left(i_{T}-n+\nu_{T}\right)+n,
\end{aligned}
$$

其中 $\mu$ 和 $t_{j}$ 为文献 [5] 中的定理 4.1 所定义的与 $k$ 无关的非负整数, 而且 $\mu \leqslant n$. 由文献 [5] 中的定理 8.3 , 对 $k \in \mathbb{N}$, 若 $\nu_{k} \neq 0$, 有

$$
\begin{gathered}
i_{k T} \geqslant k\left(i_{T}+\nu_{T}-\mu^{+}\right)+\sum_{j=1}^{\mu^{+}} 2 t_{j}^{+}+\mu^{+}-\nu_{k T}, \\
\sum_{j=1}^{\mu^{+}} 2 t_{j}^{+} \geqslant \nu_{k T}-\nu_{T}-\frac{1+(-1)^{k}}{2} \omega .
\end{gathered}
$$

从而有

$$
i_{k T} \geqslant k\left(i_{T}+\nu_{T}-n\right)+n-\nu_{T}-\frac{1+(-1)^{k}}{2} \omega,
$$

其中 $\mu^{+}, t_{j}^{+}$和 $\omega$ 是文献 [5]中的定理 8.3 所定义的与 $k$ 无关的非负整数. 由式(6)和(7), 存 在一个常数 $C$ 与 $k$ 无关, 使得总有

$$
i_{k T} \geqslant k\left(i_{T}+\nu_{T}-n\right)+C, \forall k \in \mathbb{N} .
$$

令 $k \rightarrow+\infty$, 由式(4) 与( 8 ) 得到

$$
i_{T} \leqslant \hat{i}_{T}+n-\nu_{T} .
$$

本文第二作者的文章中 ${ }^{2}$ 引进了辛道路 $\gamma$ 的 $\omega$-指标 $i_{T}(\omega)$. 其中 $\omega \in U, U$ 是复平面上的单 位圆周。其中的引理 5.1 证明了

$$
i_{T}(\omega)=i_{T}(\bar{\omega}), \forall \omega \in U .
$$

该文中的定理 5.6 证明了对足够小的正数 $\varepsilon$ 有

$$
i_{T}\left(\omega \mathrm{e}^{+\varepsilon \sqrt{-1}}\right)-i_{T}\left(\omega \mathrm{e}^{-\epsilon \sqrt{-1}}\right)=p-q, \forall \omega \in U,
$$

其中 $(p, q)$ 是 $\omega$ 作为 $\gamma(T)$ 的特征值的 Krein 型 (见文献 [6]的第一章). 注意到 $i_{T}=i_{T}(1)$, 由式(10)与 $(11)$ 有

1) Long Y. The Bott formula of the Maslov-type index theory. Preprint series of Nankai Inst of Math, No. 1996-M-001(修 改稿), 1997

2) 见 2275 页脚注 1) 


$$
\left|i_{T}(\omega)-i_{T}\right| \leqslant n \text {. }
$$

由 Long 文 $^{1)}$ 的定理 1.6 有

$$
\hat{i}_{T}=\frac{1}{2 \pi} \int_{0}^{2 \pi} i_{T}\left(\mathrm{e}^{\sqrt{-1} \theta}\right) \mathrm{d} \theta
$$

从而有

$$
\left|\hat{i}_{T}-i_{T}\right|=\left|\frac{1}{2 \pi} \int_{0}^{2 \pi}\left[i_{T}\left(\mathrm{e}^{\sqrt{-1} \theta}\right)-i_{T}\right] \mathrm{d} \theta\right| \leqslant n .
$$

由此可得

$$
\hat{i}_{T}-n \leqslant i_{T} .
$$

联立式(9)和(14), 可得

$$
\hat{i}_{T}-n \leqslant i_{T} \leqslant \hat{i}_{T}+n-\nu_{T} .
$$

用 $k T$ 代替式(15)中的 $T$, 注意到由式(4)有 $\hat{i}_{k T}=k \hat{i}_{T}$, 由此即得式(5). 证毕.

值得说明的是, 估计式 (5) 在下述意义下是最佳的估计.

例 取辛道路

$$
\gamma(t)=R\left(\frac{2 \pi t}{T}\right)^{\diamond_{n}}, t \in[0,+\infty) .
$$

这里 $R(\theta)=\left(\begin{array}{cc}\cos \theta & -\sin \theta \\ \sin \theta & \cos \theta\end{array}\right)$, 对于方块矩阵 $M_{i}=\left(\begin{array}{cc}A_{i} & B_{i} \\ C_{i} & D_{i}\end{array}\right)$, 其中 $i=1,2$, 定义

$$
M_{1} \diamond M_{2}=\left(\begin{array}{cccc}
A_{1} & 0 & B_{1} & 0 \\
0 & A_{2} & 0 & B_{2} \\
C_{1} & 0 & D_{1} & 0 \\
0 & C_{2} & 0 & D_{2}
\end{array}\right) \text {, }
$$

以及 $M^{\diamond k}$ 为 $M$ 的 $k$ 次 $\diamond-$ 积. 直接计算可得

$$
i_{k T}(\gamma)=n(2 k-1), \nu_{k T}(\gamma)=2 n, \hat{i}_{T}(\gamma)=2 n \text {. }
$$

因此有

$$
k \hat{i}_{T}(\gamma)-n=i_{k T}(\gamma)=k \hat{i}_{T}(\gamma)+n-\nu_{k T}(\gamma), \forall k \in N .
$$

估计式 (5) 对 Long 文 $^{1)}$ 中定义的辛群中由恒同矩阵出发的任意连续道路的迭代也成立. 这一 结果可以应用于 Conley 猜想的证明。

致谢 本工作为国家自然科学基金(批准号:19231201-3), 国家教委数学研究中心和求是科学 基金资助项目。

\section{参考文献}

1 Conley C, Zehnder E. Morse-type index theory for flows and periodic solutions for Hamiltonian equations. Commun Pure Appl Math, 1984, 37: 207 253

2 Long Y, Zehnder E. Morse theory for forced oscillations of asymptotically linear Hamiltonian systems. In: Albeverio S, et al. ed. Stoc Proc Phys and Geom, Singapore: World Sci, 1990, 528 563

1) 见 2275 页脚注 1) 
【研究简报】

\title{
$\mathrm{C}_{2} \mathrm{H}_{3} \mathrm{Cl}$ 的真空紫外阈值光电子谱
}

\author{
盛六四齐飞高辉俞书勤张允武 * \\ (中国科学技术大学国家问步辐射实验室、合肥 230026 。联系人)
}

\section{关键词闻值光电子能谱 同步辐射 $\mathrm{C}_{2} \mathrm{H}_{3} \mathrm{Cl}$}

同步辐射光源是优良的真空紫外光源, 以其波段广、方向性好、强度高、偏振等优点而被广 泛应用. 由于分子体系的电离阈值大部分为 $6 \sim 12 \mathrm{eV}$, 位于真空紫外波段, 所以真空紫外光 电离是研究分子电子态、超激发态、离解过程等的有力手段. 光电子谱是基于光电效应原理, 对一定能量的光子与原子、分子或固体作用后产生的电子能量进行分析. 原则上光电子谱可 以直接提供物质的所有电子无论是最强键合的还是最弱键合的结合能, 各种电离势, 原子之间 的结合能, 可以给出分子的结构. 随着同步辐射的应用, Schlag 等人 ${ }^{[1]}$ 发展了阈值光电子谱 (TPES) 技术, 它以其简单方便、得到的谱图分辨比常用的 PES 高一到两个数量级而倍受欢 迎; 与其他技术相结合, 得到了越来越广泛的应用 ${ }^{[2]}$. 阈值光电子谱也叫共振光电离谱或近 零动能电子谱. 它和普通的光电子谱 (PES) 不同之处是光子能量是变化的, 单色化的真空紫 外光作用于分子, 直接测定形成母体离子和碎片离子或发射的电子所必需的最小的能量, 接收 到的电子能量仅仅局限于接近零的电子 ${ }^{[3]}$.

阈值光电子技术可以研究分子的自电离过程, 这是其他常用方法不能做到的. 在分子的 电离阈值附近, 存在着丰富的 Rydberg 态, 这些态可以通过自电离过程而转移到较低的离子 态, 这时的直接光电离并不能给出真实的离子状态, 而且有些中性分子的某些激发态严重偏离 Franck-Condon 区域, 不能被直接光电离. 但这些态的能量与它的某些离子态能量相近, 所以 也可以通过与这些离子态的共振产生自电离. 阈值光电子与离子的高振动激发态相联系, 所 以它可以研究其他方法不能研究的内容.

阈值光电子谱在 Franck-Condon 区域内, 不但可以给出分子的绝热电离势, 还可以给出分 子的垂直电离势, 可以给出离子的各种状态, 根据这些状态的能量值和一些理论数据, 我们便 可以画出它的热能曲线.

$\mathrm{C}_{2} \mathrm{H}_{3} \mathrm{Cl}$ 分子具有较高的势稳定性, 是非常重要的化工原料, 对它的研究是很有意义的. 同时对它的实验研究可以给理论工作者提供单卤素原子与 $\pi(\mathrm{C}=\mathrm{C})$ 分子轨道的相互作用如 卤素原子的孤对电子与 $\pi(\mathrm{C}=\mathrm{C})$ 分子轨道的共振作用, 以及卤素原子的强电负性对它的影响

3 Long Y. Maslov-type index, degenerate critical points and asymptotically linear Hamiltonian systems. Science in China, Series A, 1990, 33: 1 409 1419

4 龙以明. 哈密顿系统的指标理论及其应用. 北京: 科学出版社, 1993

5 Dong D, Long Y. The iteration formula of the Maslov-type index theory with applications to nonlinear Hamiltonian systems. Trans Amer Math Soc (待发表)

6 Ekeland I. Convexity Methods in Hamiltonian Mechanics. Berlin: Springer-Verlag, 1990 